\title{
СВЕРХШИРОКОПОЛОСНАЯ ПАТЧ-АНТЕННА НАПРАВЛЕННОГО ДЕЙСТВИЯ С ИСПОЛЬЗОВАНИЕМ S-ОБРАЗНОГО МЕТАМАТЕРИАЛА
}

\author{
П. ДАВАР ${ }^{1}$, А. ДЕ ${ }^{2}$, Н. С. РАГХАВА ${ }^{2}$ \\ ${ }^{1}$ Технологический институт им. Гуру Те Бахадер, \\ Индия, Дели, Нью-Дели, 110064 \\ ${ }^{2}$ Делийский технологический университет, \\ Индия, Дели, Нью-Дели
}

\begin{abstract}
Аннотация. В работе предложена параметрическая оптимизация антенны при использовании S-образного метаматериала, встроенного в подложку антенны. При встраивании предложенной решетки из метаматериала внутрь подложки антенны ширина полосы пропускания антенны увеличивается на 74\%, а коэффициент направленности возрастает на $11 \%$. Результаты, полученные в случае моделирования в системе Ansoft HFSS на основе метода конечных элементов и программирования в среде MATLAB на основе формул САПР при использовании анализа эквивалентной схемы замещения патч-антенны, хорошо согласуются между собой. В работе поясняется эволюция S-образной формы начиная от простейшей формы разрезного кольцевого резонатора с одним кольцом. Изготовленная предлагаемая структура позволила получить отклонение от результатов моделирования не более $6 \%$. Эта антенна из метаматериала позволяет преодолеть ограничение, связанное с узкой полосой пропускания патч-антенны, и помогает поддерживать низкий профиль антенны, обеспечивая $81 \%$ миниатюризацию.
\end{abstract}

Ключевые слова: антенна; метаматериал; МТМ; материал с отрицательным коэффициентом преломления; отрицательная рефракция; RMPA; прямоугольная микрополосковая патч-антенна; DNG; HFSS; MNG

\section{1. ВВЕДЕНИЕ}

Метаматериалы (МТМ) создаются для изменения объемной магнитной проницаемости и/или диэлектрической проницаемости среды [1]. Эти материалы формируются путем периодического размещения структур, которые изменяют параметры материала, причем отдельные элементы имеют размеры меньше длины падающей электромагнитной волны. Это приводит к появлению «мета», т.е. измененного поведения, или поведения, которое недостижимо для природных материалов [2]. Форма частиц влияет на значение отрицательной диэлектрической проницаемости, соответствую- щее резонансу Фрелиха (Frohlich). Геометрия частицы c отрицательной диэлектрической проницаемостью оказывает сильное влияние на ее поверхностные плазмонные свойства.

В работе использован программный пакет HFSS, поскольку он представляет собой высокопроизводительное моделирующее устройство полного электромагнитного (ЭМ) поля для моделирования произвольных трехмерных объемных пассивных устройств [3].

В этой статье кратко изложено проектирование прямоугольной микрополосковой патч-антенны RMPA (Rectangular Microstrip Patch Antenna) с резонансной частотой 4,3 ГГц 


\section{БИБЛИОГРАФИЧЕСКИЙ СПИСОК}

1. Schantz, H. The Art and Science of Ultra-Wideband Antennas. Artech House, 2005.

2. Balanis, C. A. Antenna Theory, 4th ed. John Wiley \& Sons, Inc., 2016.

3. Pozar, D. M. "Microstrip antennas," Proc. IEEE, Vol. 80, No. 1, P. 79-91, 1992. DOI: 10.1109/5.119568.

4. Volakis, J. Antenna Engineering Handbook, 4th ed. McGraw Hill, 2007.

5. Wang. S.; Feresidis, A. P.; Goussetis, G.; Vardaxoglou, J. C. "Low-profile resonant cavity antenna with artificial magnetic conductor ground plane," Electronics Lett., Vol. 40, No. 7, P. 405-406, 2004. DOI: 10.1049/el:20040306.

6. Ahsan, M. R.; Islam, M. T.; Ullah, M. H.; Misran, N. "Bandwidth enhancement of a dual band planar monopole antenna using meandered microstrip feeding," The Scientific World J., Vol. 2014, ID 856504, 2014. DOI: 10.1155/2014/856504.

7. Lee, K. F.; Luk, K. M.; Mak, K. M.; Yang, S. L. S. "On the use of U-slots in the design of dual-and triple-band patch antennas," IEEE Antennas Propag. Mag., Vol. 53, No. 3, P. 60-74, 2011. DOI: 10.1109/MAP.2011.6028422.

8. Wong, K.-L. Compact and Broadband Microstrip Antennas. Hoboken, NJ: Wiley-Interscience, 2004.

9. Dawar, Parul; Raghava, N. S.; De, Asok. "Ultra wide band, multi-resonance antenna using swastika metamaterial," Int. J. Microwave Optical Technology, Vol. 11, No. 6, P. 423-420, 2016.

10. Ahsan, M. R.; Islam, M. T.; Ullah, M. H.; Singh, M. J.; Ali, M. T. "Metasurface reflector (MSR) loading for high performance small microstrip antenna design," PLOS One, May 2015. DOI: 10.1371/journal.pone.0127185.

11. Linden, S.; Enkrich, C.; Dolling, G.; Klein, M. W.; Zhou, J.; Koschny, T.; Soukoulis, C. M.; Burger, S.; Schmidt, F.; Wegener, M. "Photonic metamaterials: Magnetism at optical frequencies," IEEE J. Selected Topics Quantum Electronics, Vol. 12, No. 6, P. 1097-1105, 2006. DOI: 10.1109/JSTQE.2006.880600.

12. Xiong, Han; Hong, Jing-Song; Tan, Ming-Tao; Li, Bing. "Compact microstrip antenna with metamaterial for wideband applications," Turk. J. Electrical Eng. 
Comp. Sci., Vol. 21, P. 2233-2238, 2013. DOI: $\underline{10.3906 /}$ elk-1204-6.

13. Preet Kaur, Sanjay Kumar Aggarwal, Asok De," Performance enhancement of rectangular microstrip patch antenna using double $\mathrm{H}$ shaped metamaterial", Radioelectronics and Communication Journal, Vol.59,No.11,2016. 